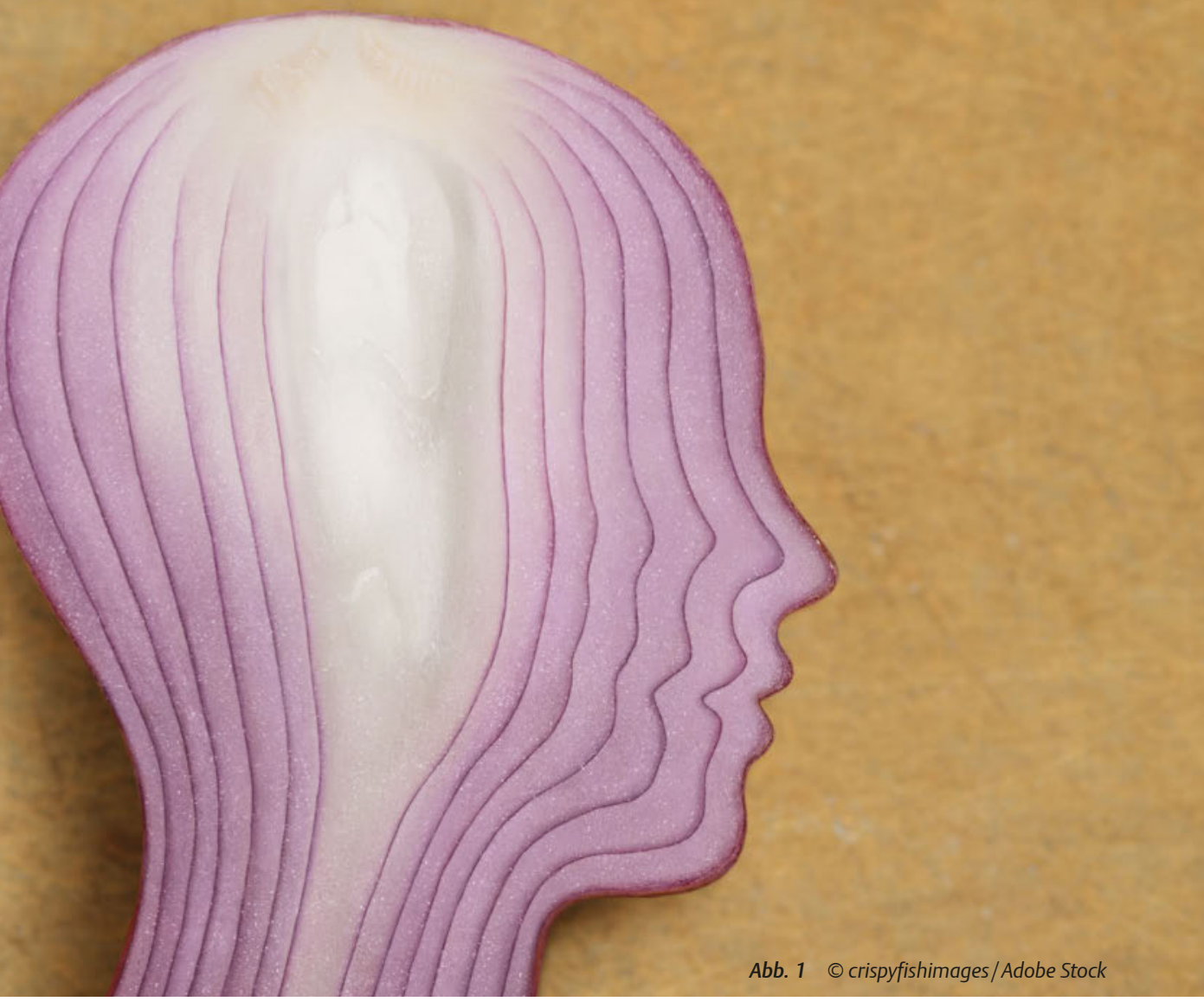

\title{
Psychologie des Fastens
}

Die Beschreibung des Fastens als hypokalorische, intermittierend oder episodisch aufgenommene Diät orientiert sich vordergründig an physiologischen Vorgängen - Doch ist das Fasten auch ein komplexer psychologischer Vorgang und zugleich ein mentaler Prozess, der gerade beim Menschen spezifische und phasenhaft verlaufende Charakteristika aufweist

Stefan Brunnhuber, Oliver Somburg

\section{Vorbemerkungen}

Fasten wird durch das bewusste Erleben einer Veränderung in der Kontrolle der Nahrungsaufnahme zu einer spezifisch menschlichen Erfahrung. Unter diesem Gesichtspunkt fasten Tiere nicht, sondern sie verfügen über die Möglichkeit, in einen hypokalorischen Modus umzuschalten, der ihnen einen Selektionsvorteil bietet. Fasten, als spezifisch menschliche Erfahrung, lässt sich als komplexes Phänomen nicht hinreichend durch den Verweis auf einzelne beteiligte Hirnregionen, neurochemische Vorgänge, durch die Darstellung der neuronalen Konnektivität oder der Darm-Gehirn-Achse abbilden. Fasten als ein Lebensvorgang bedeutet, sich aufgrund von Anpassungsmechanismen besser auf ungünstige Verhältnisse in der Umwelt einstellen zu können: die Gegebenheiten des Organismus werden optimiert und der eigene Überlebensvorteil erhöht. Darüber hinaus ist Fasten jedoch ein tiefreichender innerer Vorgang, eine innere Erfahrung, auch dann, wenn Anpassungsleistungen versagen. Otto Buchinger nannte diesen Vorgang: „Zu-sich-selberkommen“ [5], Anselm Grün [7] beschreibt ihn als etwas, bei dem „aufgedeckt wird, wer ich eigentlich bin“, der Psychologe Carl Gustav Jung nannte es letztlich „Individuation“. In anderen Heiltraditionen der Erde, wie beispielsweise den Wüstenvätern, geht es um die Überwindung der Leidenschaften und des Schattens als unbewusste Seiten des Seelenlebens. Und das Alles auch gegen die Anpassung an den gegebenen Status quo eines biologischen oder gesellschaftlichen Kontextes. Vermutlich ist das Fasten eine zivilisatorische, anthropologische Konstante wie wir es etwa von der Bewegung, dem Sprechen, Schweigen oder Schlafen her kennen.

Alle Religionen und im Kern nahezu alle langwährenden gesellschaftlichen Traditionen machen von Fastenperioden bzw. Perioden der intermittierenden Nahrungsaufnahme Gebrauch. Hingegen hat nach dem Zweiten Weltkrieg das westliche Wohlstandsmodell diese Tradition nicht wirklich weiter verfolgt. Sehr wahrscheinlich bedeutet eine intermittierende Nahrungskarenz, die bis in die strukturelle Mikroebene der Rezeptoren Veränderungen bewirkt, auch einen Überlebensvorteil gegenüber der überzivilisierten und unkritischen Nahrungsaufnahme. 


\section{Zusammenfassung}

Obwohl die physiologischen Auswirkungen der freiwilligen und kontrollierten Kalorienrestriktion bereits umfassend erforscht werden, lässt sich der komplexe Prozess des Fastens auf mentaler Ebene und als phasenhaft verlaufende Erfahrung nicht auf einzelne Hirnregionen oder Abläufe der Neurochemie reduzieren, wie sie in Konnektivitätsstudien oder Second-Brain-Studien beschrieben werden. Fasten ist eine spezifische menschliche Erfahrung, zu der die klinische Psychologie wichtige Erkenntnisse beitragen kann. Wesentliche psychische Mechanismen beim Fasten und die Auswirkungen auf das Erleben im Verlauf des Fastenvorganges, sollen hier beschrieben werden.

Zur Erklärung der Wirkung des Fastens auf die Psyche wird in den verschiedenen Fastenformen, wie Buchinger-, Mayr-, Basen-, Teilfasten, Entlastungstage etc., mehr oder weniger an der Physiologie des Fastens angesetzt, z. B. deren positive Auswirkungen auf Alterungsprozesse, beim Stressmanagement oder bei der Symptomkontrolle. Dies beschreibt aber noch nicht die dabei stattfindenden psychologischen Mechanismen.

\section{Physiologie des Fastens}

Zur Physiologie des Fastens ist beispielsweise bekannt, dass der Blutdruck fällt, die vagale Reaktionslage steigt, sich die Stressresilienz, die Neuroplastizität und der Umgang mit oxidativem Stress verbessern. Zudem wirkt Fasten antiinflammatorisch und der neurotrophe Faktor nimmt zu. Fasten hat außerdem Auswirkungen auf Endorphine, Serotonin, Ketone, die Lipolyse und Gluconeogenese, es verbessert die Insulinsensitivität, die Diurese, das IGF, Leptin, STH usw.

Diese physiologischen Aspekte sind zweifellos wichtig, für den Menschen aber relativ unspezifisch, denn sie sind ebenso bei Tieren zu finden. Sie beschreiben die Biologie des Fastens und deren teilweise viele Millionen Jahre alte Mechanismen, so wie z.B. auch die der Stressachse, welche über 300 Mio. Jahre alt ist.

\section{Fasten und Psyche}

Was passiert während des Fastens folglich unter psychologischen Gesichtspunkten, das heißt bei dem freiwilligen, bewussten und zeitlich begrenzten Verzicht auf Nahrungsmittel? Dabei geht es weder um eine Metapher, noch um eine Analogie. Fasten hat nicht nur biologisch-physiologische Dimensionen, sondern ist zugleich auch kulturell-traditioneller, psychosozialer und mental-spiritueller Natur. Unterschieden werden können: Heilfasten im Sinne der medizinischen Gesundheit; Fasten als Integration von Leib und Seele; Heilfasten als transpersonale Erfahrung im Sinne des Salis, als Gottes Segnungen oder auch Heilfasten als Heiliges, Sanctum gegenüber Säkulum, dem Weltlichen. Jedes Mal ändert sich der Blickwinkel, unter welchem der Fastenvorgang betrachtet wird und jedes Mal ist auch das zu erwartende Ergebnis ein anderes.

Die Wirkungen des Fastens auf die Psyche sind dahingehend bekannt, dass z. B. sich die Schlafqualität, die Konzentrationsfähigkeit, die Stimmung, das Schmerzerleben, die Leistungsfähigkeit, die Le- benserwartung, die Wachheit oder auch die kognitiven Reserven bei neurodegenerativen Erkrankungen verbessern. Fasten wirkt zudem anxiolytisch, antidepressiv sowie positiv auf das Körperschema. Die genannten Aspekte beschreiben die Auswirkungen des Fastens auf die Psyche. Bei vielen psychisch kranken Patienten ist die Nahrungsaufnahme in der Tat gestört. So hilft nach dem Ausgleich von krankheitsbedingten Mangelsyndromen das geführte Fasten ganz allgemein bei stressassoziierten Symptomen, bei Depression, Angst-, Trauma-, Ess- und Schlafstörungen, bei Schmerzen sowie bei Patienten, die Psychopharmaka einnehmen oder ein metabolisches Syndrom entwickelt haben. Aber das Alles beschreibt noch nicht zwangsläufig Aspekte einer „Psychologie des Fastens“ selbst.

\section{Sozialwissenschaftliche Perspektive}

Aus sozialwissenschaftlicher Perspektive ist folgende Frage von hervorzuhebendem Interesse: Was passiert, wenn Menschen in Situationen geraten, in denen Knappheit herrscht? Dabei lassen sich nun zwei Phasen unterscheiden: Zunächst werden die Effizienz und die Fokussierung erhöht, um ein Ziel unter knappen Ressourcen zu erreichen. Die Reizwahrnehmung ist intensiver, auch wenn die Reize objektiv gleichgeblieben sind. Denkschärfe und emotionale Reagibilität werden intensiviert. Hält die Knappheit allerdings länger an, entsteht in einer zweiten Phase eine bedingte Inhibition bzw. ein Tunnelblick, alles andere wird ausgeblendet. Diese Vorgänge vollziehen sich allesamt unterhalb der Wahrnehmungsschwelle von ca. $300 \mathrm{~ms}$. Menschen reagieren dann dysfunktional, machen jetzt verstärkt Fehler, das Leistungsvermögen ist eingeschränkt, die Impulsivität steigt und die Handlungskontrolle sinkt. In dieser zweiten Phase wird der Überblick verloren und die Priorisierungen von „wichtig“ und „unwichtig“ gelingen nicht mehr verlässlich.

Auf der mentalen Ebene ist dieses Fehlen von Reserven vergleichbar mit einer schlaflosen Nacht: Anstatt langfristig zu planen, werden kurzfristige Entscheidungen getroffen, der IQ fällt um über 14 Punkte und Fehler treten verstärkt auf. Messbare Effekte solcher Art sind bei nahezu jeder Form der erlebten Knappheit zu finden: bei Armut, Hunger, Zeitmangel oder dem Mangel an sozialen Kontakten.

Interessanterweise ist die menschliche Entwicklung (Entwicklung der personalen Identität, Willenssteuerung, Autonomie, Persönlichkeit usw.) auch neurobiologisch und psychologisch eng mit dem Konzept und der Wahrnehmung des Verzichts, der Hemmung, des Impulsaufschubs und der Diskriminierung verbunden. Im Gehirn finden diese differenzierten Hirntätigkeiten im Präfrontalen Cortex (PFC) statt. Die gesamte Entwicklung des PFC funktioniert letztlich über Diskriminierungsleistungen und Hemmungen. Das Gehirn filtert, bahnt und hemmt ständig: Aufmerksamkeitsspanne, Impulskontrolle, Willensbildung, Spracherwerb bis hin zur Persönlichkeitsentwicklung werden über Hemmungen und Verzicht organisiert.

Die Psychologie des Fastens erfasst weitere Seiten dieses komplexen Phänomens und es stellt sich die Frage: Welche psychologischen Mechanismen wirken beim Menschen der fastet? Wenn Fasten physiologisch als ein Neustart (Reset) aufgefasst werden kann, wie sieht dann die Psychologie dieses Resets aus? Und sind die wirkenden Mechanismen spezifisch für das Fasten? Gibt es gar abgrenzbare Phasen des Fastenvorgangs? 
Phasen einer Psychologie des Fastens

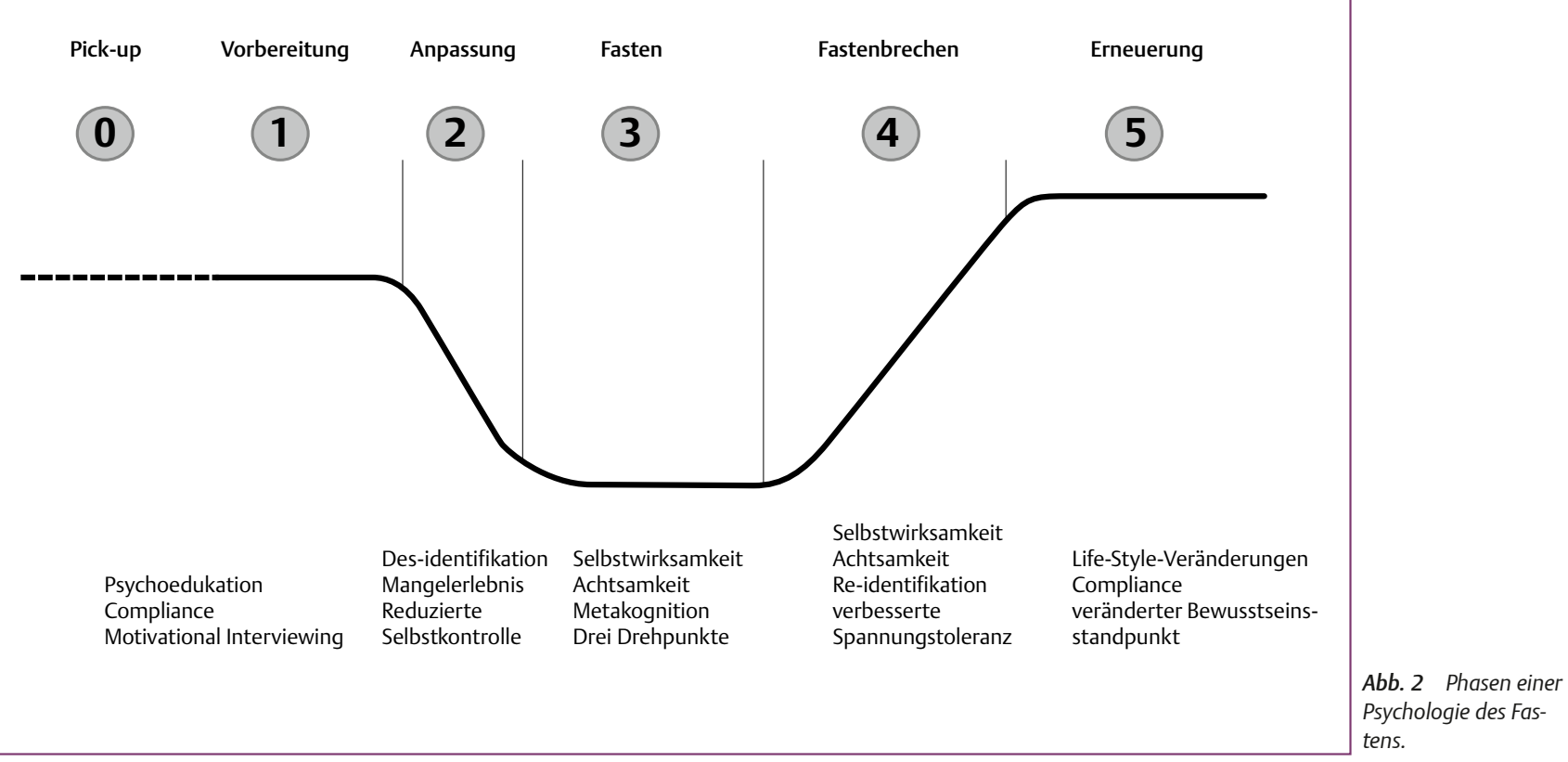

Psychologische Phasen des Fastenvorgangs

Unter psychologischen Gesichtspunkten ist es sinnvoll, den Fastenvorgang in fünf Phasen einzuteilen (Abb.2).

\section{Pick-up- Phase: Der Patient wird dort abgeholt, wo er sich befindet}

In dieser Vorphase, geht es darum, den Patienten dort abzuholen, wo er sich aktuell befindet. Vielleicht führen Verzicht und Fasten zu einer Überforderung? In dieser Phase sollen dann Ambivalenzen gegenüber dem Fasten thematisiert werden, welche sich aufgrund regulationsstarrer, stressüberlasteter Körperfunktionen- und unflexibler Ich-Funktionen ergeben können. Das Essen erfolgt hier möglicherweise überwiegend stresskompensatorisch und unachtsam.

\section{Phase 1: Klärung der Motivation und Psychoedukation}

In dieser Vorbereitungsphase stehen die Klärung der Motivation zum Fasten sowie allgemeine Informationen zum Fastenvorgang im Vordergrund. Fragen sind dann: Was soll durch das Fasten erreicht werden? Geht es um körperliche und psychische Gesundheit oder um Leistungssteigerung (Beruf, Sport, geistige Arbeit)? Oder, dient es der Selbsterfahrung oder der Überwindung von Leidenschaften und Schattenaspekten? Kurz: Warum möchte der Kandidat fasten?

Der Kandidat informiert sich dann über die Vor- und Nachteile des Fastens: kulturelle Aspekte, physiologische Auswirkungen, medizinische Belange sowie das praktische Vorgehen. In dieser Phase geht es um die Klärung der Compliance und die Vermittlung des praktischen Ablaufs. Etwa ob ein 16:8-stündiges intermittierendes Fasten, ein 2:5-tägiges oder 1-wöchiges Fasten durchgeführt werden.

Fasten ist nicht gleich Hungern. Freiwilliges Hungern ist als bewusstes Nicht-Essen noch gegen etwas gerichtet. Beim Fasten ver- schwinden schrittweise das Hungergefühl und das Bedürfnis etwas zu essen. Es besteht kein Wunsch nach einer kalorischen Nahrungsaufnahme.

Mit einfachen Techniken der antwortentwickelnden Selbstexploration (Motivational Interviewing) lassen sich wirkungsvoll Ambivalenzen auflösen, Änderungsbereitschaft und eine intrinsische Motivation zur Verhaltensänderung herstellen. Denn es gilt: Kein Mensch ist nicht nicht motiviert [9].

Fasten sollte von Anfang an ebenso geistige und intellektuelle Funktionen einbeziehen, d.h.: weniger sprechen und nachdenken, mehr kontemplativ als aktiv sein. Gedanken können selbst sättigend sein und das Fasten wird dann schnell instrumentalisiert. Geistiges Fasten dagegen ist eine Form der mentalen Enthaltsamkeit (Dulcedo Dei = Süssigkeit Gottes). Klinisch vertiefen sich Fasten und Stille häufig gegenseitig, dabei entsteht ein veränderter Bewusstseinsschwerpunkt.

Der Fastenvorgang ist psychologisch einerseits zwar eine persönliche Erfahrung, aber kein Selbstzweck, sondern ein Mittel, um etwas an sich zu erkennen oder zu verändern. In diesem Sinne vermittelt das Fasten Einsicht bzw. Einblick in die unsichtbare Vernetzung mit der Natur und der sozialen Umwelt und die Realisierung der wechselseitigen Abhängigkeit und Verwobenheit. Daraus kann sich dann eine verstärkte Solidarität mit anderen entwickeln oder auch die zweckgerichtete Motivation zum politischen Fasten entstehen. Allgemein kann man sicher sagen: Die Motivation zum Fasten bestimmt den gesamten Fastenverlauf.

Beispiel für eine ungünstige Motivation ist eine versteckte narzisstische Leistungspathologie mit uneingestandenen Optimierungsabsichten, wodurch dann unausgesprochene Ängste kompensiert werden. 
Günstige Motivationen hingegen sind das Fasten als kohärentes mentales Instrument zur Veränderung und Umwandlung des Bewusstseinsschwerpunktes, zur Distanzierung von Ich-Funktionen und dem Wunsch der Einbettung in einen größeren Gesamtzusammenhang.

\section{Phase 2: Anpassungsvorgang}

Diese Phase ist gekennzeichnet durch das innere „Los-lassen“ von Gewohnheiten und Automatismen, der Rücknahme von täglichen Ritualen und Beginn des Umschaltens von Körperfunktionen. Dadurch kommt es zu einer fortschreitenden Des-Identifikation von Grobstofflichem, einer reduzierten Selbstkontrolle und dem Erleben von Mangel / Knappheit, bedingter Inhibition, Leistungsabfall, reduzierter Spannungstoleranz und Selbstwirksamkeit. Diese Anpassungsphase wird häufig als kritisch und unstabil erlebt, stellt aber eine wichtige Voraussetzung für den weiteren Verlauf dar.

\section{Phase 3: Fasten als Selbstwirksamkeit und Des-Identifikation}

Charakteristisch in dieser Phase sind sogenannte Selbstwirksamkeitserwartungen [1]: die subjektive Gewissheit neue und schwierige Anforderungen selbst bewältigen zu können.

Das Konzept spielt auch in der Psychotherapie eine zentrale Rolle und umfasst folgende Aspekte:

- die subjektive Gewissheit etwas gemeistert zu haben (Ich-

Funktionen),

- die internale Kontrolle (keine Willkür, Glück, Zufall oder Belie-

bigkeit, sondern Resultat des Ich),

- die Erfahrung wird bei anderen beobachtet, gelernt und unterstützt (Peer-Group-Erfahrung),

- körperliche und emotionale Vorgänge werden dabei positiv interpretiert.

Die Selbstwirksamkeitserwartung bildet eine stabile Persönlichkeitsvariable mit einem hohen Vorhersagewert (ASF: Aachener Selbstwirksamkeit Fragebogen). Sie ist gekoppelt mit der Fähigkeit zur internalen Kontrolle. Unzureichende internale Kontrolle wiederum korreliert mit zahlreichen psychischen und psychosomatischen Erkrankungen (Angst, Depression, Sucht, Schmerz, Neurotizismus und fehlende Resilienz).

Neben der Selbstwirksamkeit kommt es zu einem anhaltenden „inneren Los-lassen“ (Des-Identifikation): Identifikationen stellen in der Psychologie die Voraussetzungen für den Aufbau von IchFunktionen dar, dienen der Internalisierung von Objektbeziehungen und dem Aufbau einer stabilen Selbstrepräsentanz (experimentelle Identifikation z. B. im Psychodrama oder Rollenspiel). Jedes „innere Los-lassen“ (Des-Identifikation) schafft so die Voraussetzungen für einen Perspektivenwechsel und verläuft in der Regel über drei Stufen:

- Des-Identifikation mit der Anatomie und dem Sensorium (begriffliche Form des Hungers, des Gewichts und des Essens),

- Des-Identifikation mit Emotionen und Denken (Repräsentationen von Leistungen und biographischen Ereignissen) und

- Des-Identifikation mit Ego und Ich-Funktionen.

Bei jedem Schritt der Des-Identifikation (körperlich, emotional, gedanklich, Ich-Funktion) entsteht zunehmend ein Zeugenzustand bzw. eine Metakognition. Der Vorgang wird distanziert betrachtet und der Betrachter identifiziert sich nicht mehr mit dem Geschehen. Die innere und äußere Welt wird intensiver wahrgenommen und bleibt dennoch weniger vereinnahmend (affiziert). Im letzten Stadium geht auch die Selbstwirksamkeit so weit zurück, dass in Leere, Stille und absoluter Achtsamkeit postkonventionelle oder transpersonale Affekte, die nicht mehr mit den Ich-Funktionen erzeugt werden können, klinisch vordergründig werden. Hierzu zählen etwa Gnade, Demut, Dankbarkeit, absichtslose Empathie und Mitgefühl. Diese können in der Fremd- und Selbstwahrnehmung erfahren werden. Beide - Selbstwirksamkeit und Des-Identifikation wechseln sich auf jeder der Ebenen immer wieder aufs Neue ab: regressiv oder progressiv.

Warum ist das für das Fasten wichtig? Weil das Ausmaß an „innerem Los-lassen“ (Des-Identifikation), das Ausmaß der essenziellen Erfahrung und des Selbsterkennens und infolgedessen auch die Compliance für Lebensstilmodifikationen wesentlich bestimmen werden. Das heißt, die ursprüngliche Motivation (Gesundheit, Leistung) leitet die Tiefe der Des-Identifikation ein, die wiederum im weiteren Verlauf des Fastenvorgangs bestimmt, aus welchem Bewusstseinsschwerpunkt die Welt wahrgenommen und darauf reagiert wird.

\section{Phase 4: Fastenbrechen oder Re-feeding}

In dieser Phase wird der umgekehrte Weg durchlaufen: die Re-Identifikation mit den eigenen Gedanken, Emotionen und dem Körper, den Sozialkontakten sowie die Wiedergewinnung von bewusster Kontrolle.

\section{Phase 5: Erneuerung}

Idealtypisch kommt es durch die psychologisch wirksamen Mechanismen und nicht zwangsläufig durch die Physiologie des Fastens zu nachhaltigen Änderungen des Lebensstils und der Compliance. Klinisch gilt die Regel, dass das Ausmaß an Des-Identifikation und die damit einhergehenden positiven Erfahrungen, die langfristige Adhärenz bestimmen werden.

Sehr wahrscheinlich werden regelmäßig beim Fasten diese 5 Phasen psychologisch durchlaufen und gehen ineinander über. Die einzelnen psychologischen Mechanismen sind individuell unterschiedlich intensiv wirksam (Psychoedukation, Des-Identifikation,

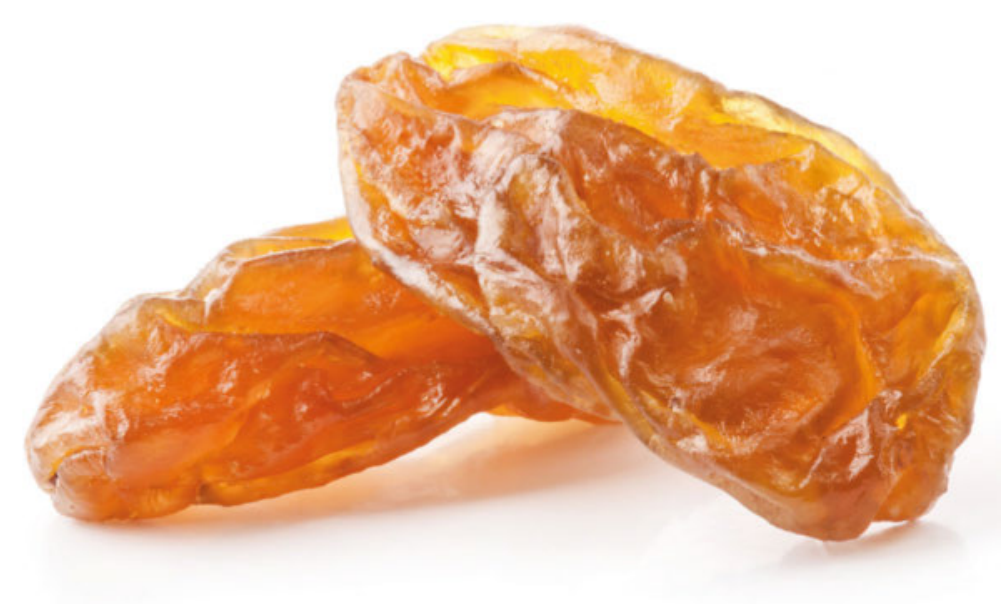

Abb. 3 Rosinenübung: Die schrittweise sensorische Erfahrung unterstützt den achtsamen Umgang mit Nahrungsmitteln. ( ) msk.nina/Adobe Stock 
Psychologie des Mangels, Kontrollverlust, Selbstwirksamkeitserwartungen, Metakognition, Achtsamkeit, Auftauchen postkonventioneller Affekte). Klinisch ist es dabei weniger entscheidend, wie lange gefastet wird, sondern dass die dabei stattfindenden psychischen Vorgänge aus sich heraus entstehen, vertrauend und selbstleitend zugelassen und richtig zugeordnet werden können.

\section{Mögliche Umsetzung in einem stationären Setting}

Während die meisten Mindfulness-Based-Programme einen linearen und modularen Aufbau verfolgen, in dem klinische Symptome und Beschwerden im Vordergrund stehen, ist der stufenartige Ansatz vorteilhafter (Abb.4). Praktisch gewährleistet ein Stufenprotokoll bei allen Patienten eine höhere Compliance und Adhärenz im Umgang mit der Nahrungsaufnahme. Die Stufen bauen aufeinander auf und ergeben dabei unterschiedliche Lebensstilmodifikation. In der Regel umfassen die späteren Stufen die früheren. Das Programm besteht aus 5 Stufen und ist nicht modular, sondern integrativ und transformativ.

\section{Fünf Stufen eines Mindful-Based-Eating \& Fasting Basis-} programms (MB-E\&F)

Stufe 0: MBM-E \& F Einführung

Fragebogen zu Ernährungsgewohnheiten oder auch der sog. Mindfullness-Eating-Zirkel.

\section{Stufe 1: MBM-E \& F Psychoedukation}

Diese Stufe hat informativen Charakter. Der Fokus liegt auf selbstwirksamen Erfahrungen wie z. B. Spannungstoleranz (Fasten erhöht die Fähigkeit der Latenz zwischen Reiz und Reaktion. Dies ist z.B. bei Suchterkrankungen erheblich gestört), Informationen über die Vorteile von Bio- und pflanzlicher Kost. Auf dieser Stufe gilt es, den Zusammenhang zwischen Appetit und Hunger zu klären. Erkenntnis über den erforderlichen Nährstoffbedarf und die Auswirkungen von Energiemangel, die Rolle von Langeweile und Ess-Angewohnheiten sowie des kognitiven Einflusses auf das Essverhalten gehören ebenfalls in diese Stufe.

Dazu gehört die Vermittlung der 7 Formen des Hungers:

- Augen-Hunger (visuell),

- Nase-Hunger (Geruch),

- Gaumen-Hunger (haptisch),

- Magen-Hunger (konventionell, knurrt, Rhythmus der gewohnten Nahrungsaufnahme),

- geistiger Hunger (Ideen, Wissen über Ernährung),

- Leibgericht-Hunger („Herz-Emotionen“),

- zellulärer Hunger (eigentlicher Hunger als physiologisches Erfordernis).

Hilfreiche Instrumente in der praktischen Umsetzung können z.B. sein: das Riechen an Nahrungsmitteln, das Einüben der verschiedenen Formen des Hungers; ein achtsamer Umgang mit Nahrungsmitteln durch eine schrittweise sensorischen Erfahrung („Rosinenübung“).

Vermittlung durch Psychologen, Pflege

Stufe 2: MBM-E \& F Achtsamkeit

Achtsam zu essen bedeutet langsamer (jeden Bissen 20-mal kauen), bewusster, schweigend und ritualisiert, allein oder in der Gruppe zu

MB-E\&F: Stufenprogramm

Mindful-Based-Essen \& Fasten

MB-E\&F Vollfasten (Stufe 5):

3-7 Tage, $100-350 \mathrm{kcal} / \mathrm{d}$

MB-E\&F Entlastungstage (Stufe 4):

$250 \mathrm{kcal} / \mathrm{d}$;

1-2 mal pro Woche:

erweiterte Verhaltensänderung, z.B.

,Reis-Tage', ,Entschlackungs-Tage

MB-E\&F Selektive Diät (Stufe 3):

hypokalorisch, Weglassen bestimmter Nahrungsmittel/Getränke

(Alkohol, Softdrinks, Zucker) oder spezielle Diäten:

eingeschränkte Verhaltensänderungen

MB-E\&F Achtsamkeit (Stufe 2): langsames (20-mal kauen),

bewusstes, ritualisiertes Essen oder Essen im Schweigen, einzeln, in der Gruppe,

Achtsamkeitsaspekt, ohne Veränderung der Diät

MB-E\&F Psychoedukation (Stufe 1): (7 Formen des Hungers), Rosinenübung,

Umgang mit Nahrungsmitteln, Rituale des Essens - Spannungstoleranz, Bio-Kost, Fragebogen, kognitiver Anteil

MB-E\&F Einführung (Stufe 0):

Fragebogen zu Ernährungsgewohnheiten oder auch der sog. Mindfullness-Eating-Zirkel

Abb. 4 Stufen des Mindful-BasedEating \& Fasting Basisprogramms (MB-E\&F). 
essen. In dieser Stufe wird die Diät noch nicht verändert. Vermittlung durch Psychologen, Pflege

\section{Stufe 3: MBM-E \& F Selektive Diät}

In diesem Modul wird mit einer veränderten Nahrungsaufnahme begonnen: Sie kann hypokalorisch sein oder den Verzicht auf bestimmte Nahrungsmittel/Getränke beinhalten (z. B. Alkohol, Softdrinks, Zucker) oder in einer speziellen Diät (Reis-Tag, Entschlackungs-Tag) bestehen. Damit wird eine gezielte Verhaltensänderung angestrebt.

Vermittlung durch Ärzte, Pflege

\section{Stufe 4: MBM-E \& F Entlastungstage}

Die Entlastungstage können eine erweiterte Verhaltensänderung bewirken. Es wird 1- bis 2-mal pro Woche ein Entlastungstag eingelegt, an dem 250-350 kcal / Tag aufgenommen werden. Oder spezifische „Reis-Tage“ etc.

Vermittlung durch Ärzte, Pflege

\section{Stufe 5: MBM-E \& F Medizinisches Vollfastenprogramm}

Das Vollfastenprogramm nach Buchinger dauert mindestens 3, maximal 7 Tage. Erlaubt sind 100-350 kcal pro Tag in Form von Suppen und / oder Fruchtsaft sowie reichlich Flüssigkeit (>2-3 Liter Tee und Wasser). Während des Fastens erfolgen Laborwertkontrollen, ausleitende Verfahren (Einläufe) sowie die Kontrolle von Blutdruck und Puls, ggf. muss die Einnahme verordneter Medikamente angepasst werden.

Vermittlung und Überwachung durch Ärzte, Pflege

\section{Fazit}

Fasten ist mit dem Verzehr einer hypokalorischen Diät, der Intervallverlängerung zwischen zwei Mahlzeiten oder episodisch umschriebenem Nahrungsverzicht vorwiegend als physiologischer Vorgang beschrieben. Die beim Fastenprozess wirkenden psychologischen Mechanismen werden dabei nicht sichtbar. Die für den Fastenvorgang spezifischen Mechanismen lassen sich in Form von mentalen Änderungsprozessen auch in der Alltagspsychologie und der Psychopathologie wiederfinden.

Charakteristisch dennoch für die psychologischen Vorgänge beim Fasten sind das Zusammen- und Wechselspiel von Des-Identifikation (,vollständiges inneres Loslassen“) und leiblicher Selbstwirksamkeit in komplementärer Form, welches eine andere Ebene der Erfahrung eröffnet, die letztlich transformativ wirken kann. Diese Form der Komplementärbeziehung verweist auf elementare und lebendige Wirkprinzipien innerhalb der menschlichen Psyche im Allgemeinen.

Fasten als spezifische und komplexe menschliche Erfahrung lässt sich nicht auf Hirnregionen oder neurochemische Vorgänge reduzieren, wie der Eindruck aus Konnektivitätsstudien und Darm-HirnStudien entstehen kann. Im Gegensatz zu neocartesianischen Denkweisen mit der Trennung von Geist und Körper haben fernöstliche Herangehensweisen mit bipolaren, komplementären, bidirektionalen Prinzipien einen weiterführenden Ansatz mit der Kraft von bewusstem Verzicht-, Deprivations- oder Mangelerlebnissen. Das ist wahrscheinlich beim Schweigen oder Meditieren als tiefreichende innere Erfahrungen nicht anders.

Erst das Zusammenspiel von physiologischen und psychologischen Wirkfaktoren jedoch macht aus dem Fastenvorgang ein spe- zifisches, individuell ideograhisches und persönliches Ereignis. Jeder der ernsthaft fastet, kann diese Mechanismen mehr oder weniger bei sich feststellen.

Erst wenn die psychologischen gemeinsam mit den physiologischen Mechanismen wirken, wird die spezifisch menschliche Aktivität des Fastens erlebbar und zu einer essenziellen Erfahrung.

\section{Drei wesentliche psychologische Drehpunkte beim Fastenvorgang}

1) Identifikation/Des-Identifikation mit körperlichen Vorgängen Prozesshafte Distanzierung von grobstofflichen, sensomotorischen und physiologischen körperlichen Vorgängen. Der Fastende bleibt mit Emotionen und Kognitionen aber weiterhin identifiziert. Auf diese Weise gelingt ihm die Erfahrung der Selbstwirksamkeit. Der Kern bleibt in seinen formalen Ich-Funktionen erhalten (Ego).

2) Identifikation/Des-Identifikation mit Gedanken und Affekten Dabei geht es um die prozesshafte Distanzierung von autobiographischen Bezügen, Lebenszielen, Sozialkontakten, Sinnentwürfen, Schicksalsschlägen - Themen, die auch Gegenstand einer Psychotherapie sind.

3) Identifikation/Des-Identifikation mit dem Ego/den Ich-Funktionen selbst

Auf diese Weise entstehen andere, nicht mehr Ich-geleitete Affektprofile, sog. transpersonale Affekte: Demut, Dankbarkeit, Vergebung, das tiefreichende Gefühl der sozialen Vernetztheit mit anderen Menschen, ein universelles Gewahrsein sowie Güte; aus konkretem Mitgefühl entsteht tief verinnerlichte absichtslose Empathie gegenüber allen Wesen. Auf diesem Drehpunkt kann zwischen relativen und absoluten Affekten unterschieden werden:

- relative Affekte (skalenabhängig): mehr oder weniger Freude oder Ärger als Gestimmtheit

- absolute Affekte (skalenunabhängig): universal, global, ewig, zeitlos, transpersonal

Die aufgeführten Punkte können klinisch erfasst und registriert werden. Dabei sind weniger die kognitiven Inhalte der Fastenerfahrung, sondern die dabei geäußerten Affektprofile wesentlich. Die drei Drehpunkte ergeben Hinweise auf die zu erwartende Compliance und inwieweit das Fasten transfomativ auf den Lebensstil wirken kann. Der Grad des erreichten „inneren Los-lassens“ vollzieht die Tiefe der entstandenen Erfahrung und das Erneuerungsgefühl.

Compliance, initiierte Lebensstilmodifikationen und Transformationen im Nachgang einer Fastenkur hängen somit vom Ausmaß des Durchlaufens der jeweiligen Drehpunkte ab. Je klarer die initiale Motivationslage und je umfassender das Wechselspiel von „innerem Los-lassen“ (Des-Identifikation) und leiblicher Selbstwirksamkeit sind, desto umfassender und stärker werden die Compliance und die Transformation ausfallen. Die Übertragung dieser grundmenschlichen Erfahrungen auf andere anthropologische Konstanten wie z.B. Sprechen und Schweigen, Bewegung und Ruhe kann ähnliche transformative innere Anteile erreichen, was ebenfalls vor allem in verschiedenen Religionen und Kulturen bereits nachhaltig praktiziert wird.

Interessenkonflikt: Die Autoren erklären, dass kein Interessenkonflikt besteht.

Online zu finden unter

http://dx.doi.org/10.1055/a-0572-8367 


\section{Literatur}

$\overline{1}$ Bandura A. Self-efficacy. The exercise of control. New York: W. H. Freeman; 1997

$\overline{2}$ Bays CJ. Mindful Eating. A guide to Rediscovering a healthy and joyful relationship with Food. Boston London: Shamhala; 2009

$\overline{3}$ Brantschen N. Fasten für Körper, Geist und Seele. Freiburg: Aira; 2006

$\overline{4}$ Borchmann D. Fasting, Restrained Eating and cognitive Performance. A Literatur Review from 1998-2006 [Diplomarbeit]. Universität Hamburg: 2007

$\overline{5}$ Buchinger O. Das Heilfasten. 1. Aufl. Stuttgart: Hippokrates; 1935

$\overline{6}$ Brunnhuber S. Die Kunst der Transformation. Freiburg: Herder; 2016

$\overline{7}$ Grün A. Meine Innere Quelle finden. Münsterschwarzach: Vier Türme Verlag; 2015

8 Korn LE. Nutrition Essentials for mental Health. New York: Norton; 2016

9 Miller W, Rollnick S. Motivierende Gesprächsführung. Freiburg: Lambertus; 2015

$\overline{10}$ Ostafin BD, Robinson MD, Meier BP, eds. Handbook of Mindfulness and Self Regulation. Springer; 2015

$\overline{11}$ Tucker $\mathrm{T}$. The great starvation experiment Ancel Key and the men who starved for science. Minneapolis: University Press; 2007

$\overline{12}$ Wanskink B. Mindless Eating. Why we eat more then we think. New York: Bantam; 2006

$\overline{13}$ Wilhelmi de Toledo F. Buchinger Heilfasten. Stuttgart: Trias; 2003

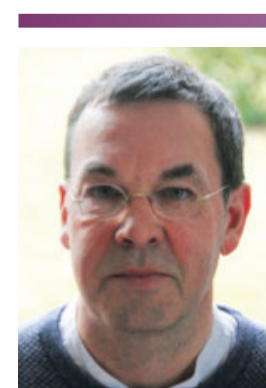

\section{Dr. med. Oliver Somburg}

Chefarzt der Klinik für Psychiatrie, Psychosomatik und Psychotherapie des Diakoniewerks Zschadraß

Im Park $2 b$

04680 Colditz

Oliver Somburg ist Chefarzt der Klinik für Psychiatrie, Psychotherapie und Psychosomatik des Diakoniewerks Zschadraß. Er ist Facharzt für Psychiatrie und Psychotherapie und Facharzt für Neurologie. Interessenschwerpunkte sind Humanistische Psychotherapie, Mind-Body-Medizin, Ernährung und Fasten.

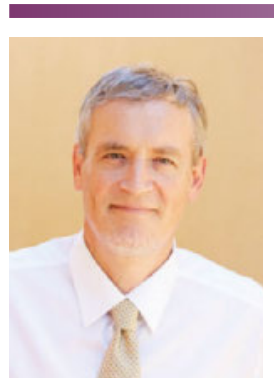

Prof. Dr. med. habil. Dr. rer. soc MA phil. Stefan Brunnhuber Diakonie Kliniken Zschadraß

Klinik und Poliklinik für Integrative Psychiatrie

Psychosomatik und Psychotherapie

Im Park $15 \mathrm{~A}$

04680 Colditz / Zschadraß

brunnhuber.cor@gmxpro.de

Stefan Brunnhuber ist Ärztlicher Direktor und Chefarzt der Diakonie Kliniken für Integrative Psychiatrie und Psychosomatik in Zschadraß; W-3 Professur für Psychosomatik und Naturheilkunde; langjährige klinische und persönliche Erfahrungen im Bereich des intermittierenden Fastens, Aufenthalt bei den Wüstenvätern, ZEN und Yoga; www.stefan-brunnhuber.de 